\title{
Bilirubin directly disrupts membrane lipid polarity and fluidity, protein order, and redox status in rat mitochondria
}

\author{
Cecília M.P. Rodrigues ${ }^{1, *}$, Susana Solá ${ }^{1}$, Maria A. Brito ${ }^{1}$, Dora Brites ${ }^{1}$, José J.G. Moura ${ }^{2}$ \\ ${ }^{1}$ Centro de Patogénese Molecular, Faculdade de Farmácia, University of Lisbon, Av. Forças Armadas, 1600-083 Lisbon, Portugal \\ ${ }^{2}$ Departamento de Química, Faculdade de Ciências e Tecnologia, Universidade Nova de Lisboa, 2825-114 Monte de Caparica, Portugal
}

Background/Aims: Unconjugated bilirubin (UCB) impairs crucial aspects of cell function and induces apoptosis in primary cultured neurones. While mechanisms of cytotoxicity begin to unfold, mitochondria appear as potential primary targets.

Methods: We used electron paramagnetic resonance spectroscopy analysis of isolated rat mitochondria to test the hypothesis that UCB physically interacts with mitochondria to induce structural membrane perturbation, leading to increased permeability, and subsequent release of apoptotic factors.

Results: Our data demonstrate profound changes on mitochondrial membrane properties during incubation with UCB, including modified membrane lipid polarity and fluidity $(P<0.01)$, as well as disrupted protein mobility $(P<0.001)$. Consistent with increased permeability, cytochrome $c$ was released from the intermembrane space $(P<0.01)$, perhaps uncoupling the respiratory chain and further increasing oxidative stress $(P<0.01)$. Both ursodeoxycholate, a mitochondrial-membrane stabilising agent, and cyclosporine $\mathrm{A}$, an inhibitor of the permeability transition, almost completely abrogated UCB-induced perturbation.

Conclusions: UCB directly interacts with mitochondria influencing membrane lipid and protein properties, redox status, and cytochrome $c$ content. Thus, apoptosis induced by UCB may be mediated, at least in part, by physical perturbation of the mitochondrial membrane. These novel findings should ultimately prove useful to our evolving understanding of UCB cytotoxicity.

(C) 2002 European Association for the Study of the Liver. Published by Elsevier Science B.V. All rights reserved.

Keywords: Bilirubin cytotoxicity; Cytochrome $c$; Electron paramagnetic resonance spectroscopy; Mitochondrial membrane structure

\section{Introduction}

Accumulation of unconjugated bilirubin (UCB) in the central nervous system contributes to cell damage during severe neonatal hyperbilirubinemia and Crigler-Najjar type I syndrome leading to bilirubin encephalopathy $[1,2]$. In fact, numerous crucial aspects of cell function are impaired by UCB [3-11], perhaps resulting in apoptotic processes [11-13]. Though primary targets are still to be identified, mitochondria have long ago been described as particularly vulnerable to UCB-mediated toxicity [14-18].

Received 9 May 2001; received in revised form 5 October 2001; accepted 8 November 2001

* Corresponding author. Tel.: +351-21-794-6400; fax: +351-21-7946491.

E-mail address: cmprodrigues@ff.ul.pt (C.M.P. Rodrigues).
To this end, we have recently demonstrated that exposure of isolated mitochondria to UCB resulted in swelling and mitochondrial membrane permeabilisation [12]. Both cyclosporine $\mathrm{A}$, a known inhibitor of the megapore channel, and ursodeoxycholate, which appears to exhibit an ubiquitous protective effect involving the mitochondrial membrane [19-21], prevented UCB-induced alterations. Finally, ursodeoxycholate, also shown to protect from apoptosis by modulating mitochondrial alterations, equally inhibited UCB-induced toxicity in nerve cells $[12,13]$. Thus, mitochondria appear to mediate apoptosis by UCB, but the underlying molecular mechanism(s) of toxicity remains poorly understood.

Two major execution points in mammalian apoptotic cell death involve caspase activation and mitochondrial dysfunction [22,23]. Mitochondrial perturbation includes 
opening of the permeability transition pore, and is influenced by multiple parameters, many of which induce apoptosis. These include collapse of mitochondrial transmembrane potential, production of reactive oxygen species, and release of cytochrome $c$ from the intermembrane space, resulting in self-amplifying cascades. The molecular mechanism(s) for release of caspase-activating proteins from the mitochondria into the cytosol remains controversial, but may involve direct interaction of the apoptotic agent with the mitochondrial membrane.

In the present study, we used electron paramagnetic resonance (EPR) spectroscopy analyses of isolated mitochondria to test the hypothesis that UCB acts locally in mitochondrial membranes to induce structural perturbation, leading to the release of apoptotic factors. The results demonstrate profound changes on mitochondrial membrane properties during incubation with UCB, resulting in disrupted membrane lipid polarity and order, as well as modified protein mobility. As a consequence of altered mitochondrial membrane permeability, cytochrome $c$ is released, perhaps inhibiting electron transfer in the respiratory chain and further increasing reactive oxygen species production. Thus, apoptotic cell death induced by UCB may be mediated, in part, by its effect of perturbing mitochondrial membranes.

\section{Materials and methods}

\subsection{Mitochondria isolation}

Low calcium brain and liver mitochondria were isolated from adult Wistar rats as previously described [24-26]. Following isolation, mitochondria were maintained on ice and used within $3 \mathrm{~h}$ of isolation. Protein concentrations were determined using the Bio-Rad protein assay (BioRad Laboratories, Hercules, CA, USA). All animals received humane care and the study protocol complied with the institution's guidelines.

\subsection{EPR spectroscopy and spin-labelling techniques}

Changes in membrane structure can be measured by EPR spectroscopy using paramagnetic reporter groups incorporated into membranes. The polarity of mitochondrial membranes was examined at varying depths with doxyl stearic acid spin labels (5-, 7-, and 16-DSA) (Sigma Chemical Co., St. Louis, MO, USA), which contain a nitroxide group at different positions along the hydrocarbon chain of the stearic acid molecule [2729]. In the 5-DSA probe, the paramagnetic centre resides near the lipidwater interface, while in the 16-DSA localises deeper in the lipid bilayer. A reliable parameter of the environment of these probes, calculated from direct measurements of the parallel and perpendicular components of the hyperfine tensor of the spin label, is the isotropic splitting factor $a_{0}$. A high $a_{0}$ reflects increased polarity of the membrane. In addition, alterations in membrane dynamic properties of 5- and 7-DSA probes, showing restricted motion in the membrane, were evaluated by measuring the outer half-width at half-height of the low-field extremum $(\Delta l)$. The larger $\Delta l$, the more motion and less order in the local microenvironment reported by the nitroxide group. For the 16-DSA spin label, which showed a higher degree of motional freedom, the ratio of the height of the low-field and the centrefield line $\left(h_{+1} / h_{0}\right)$ was used as an empirical measurement of membrane lipid organisation [30,31]. Finally, we tested the 4-maleimido-2,2,6,6-tetramethylpiperidinooxyl (4-maleimido-TEMPO) label (Sigma Chemical
Co.), which binds to the sulfhydril group of proteins giving information about mobility of protein reactive groups and allowing measurement of changes in structure of protein-rich membranes at the surface level $[29,32,33]$. The ratio of the height of the midline to the height of the high-field line of the spectrum $\left(h_{0} / h_{-1}\right)$ reflects the freedom of motion of this probe at its binding site. A high ratio indicates a low freedom of motion.

All spin labels were dissolved in chloroform, separated into 1- $\mu \mathrm{g}$ aliquots, evaporated under nitrogen, and left under vacuum for $2 \mathrm{~h}$. Freshly isolated intact mitochondria (100-150 $\mu \mathrm{g}$ protein) were incubated in spinlabel-coated tubes with gentle shaking, for $90 \mathrm{~min}$ at $37^{\circ} \mathrm{C},(5-, 7-, 16-$ DSA), or for $1 \mathrm{~min}$ at $22^{\circ} \mathrm{C}$ (4-maleimido-TEMPO), yielding systems containing $5 \mathrm{~mol}$ spin label per $100 \mathrm{~mol}$ membrane lipids ( 5 mol.\% probe).

Mitochondrial membrane lipid peroxidation was also assessed using EPR as described previously [34-36]. In brief, freshly isolated mitochondria (5$10 \mu \mathrm{g}$ of protein) were incubated with two membrane-associated, oxidation-sensitive, paramagnetic probes (1- $\mu$ g aliquots), 5- and 12-DSA, with gentle agitation for $20 \mathrm{~min}$ at $22^{\circ} \mathrm{C}$. Increased production of reactive oxygen species results in loss of paramagnetism of the probe, and hence loss of signal amplitude, measured as the height of the centre-field line of the spectrum.

Labelled mitochondrial membranes were then exposed to UCB $(0.9,4.3$, and $8.6 \mu \mathrm{M}$ ) (Sigma Chemical Co.) in Chelex-100-treated respiration buffer $(10 \mathrm{mM}$ HEPES, $10 \mathrm{mM}$ succinate, $215 \mathrm{mM}$ mannitol, $71 \mathrm{mM}$ sucrose, $\mathrm{pH} \mathrm{7.4)} \mathrm{for} 5 \mathrm{~min}$ at $22^{\circ} \mathrm{C}$, yielding a molar ratio of approximately $2 \mathrm{~mol} \mathrm{UCB}$ to $100 \mathrm{~mol}$ endogenous lipids. UCB concentrations were selected based on the general believe that toxicity is cumulative and dependent on concentration and exposure time. Thus, greater concentrations are necessary to evaluate toxicity after short incubation periods required by the instability of the molecule [37]. In co-incubation studies, labelled mitochondria were pre-treated with either ursodeoxycholate $(500 \mu \mathrm{M})$ or cyclosporine A $(5 \mu \mathrm{M})$ (Sigma Chemical Co.) for $5 \mathrm{~min}$, which remained in the incubation medium. All studies used purified UCB [38] that was dissolved in dimethylsulfoxide ( $0.86 \mathrm{mM}$ stock solution), immediately prior to use, and kept under light protection throughout the experiment. Although never exceeding 1\%, similar concentrations of dimethylsulfoxide were added to relevant controls. After incubations, the mitochondrial suspension was spun down at $12000 \times g$ for $3 \mathrm{~min}$, and the pellet resuspended in respiration buffer $(60 \mu \mathrm{l})$. Samples were then sucked into glass capillaries, sealed at both ends, and introduced in standard 4-mm quartz tubes containing silicone oil for thermal stability. All spectra were acquired at $9.8 \mathrm{GHz}$ (X-band) on a Bruker EMX EPR spectrometer (Bruker, Karlsruhe, Germany) using a rectangular cavity (model ER 4102ST) and 100 $\mathrm{kHz}$ field modulation frequency, $1.05 \mathrm{G}$ modulation amplitude and $20 \mathrm{~mW}$ microwave power, at $22^{\circ} \mathrm{C}$.

\subsection{Determination of cytochrome $c$ content in supernatants and mitochondrial pellets}

Following incubations, mitochondria were centrifuged at $12000 \times g$ for $3 \mathrm{~min}$ at $4^{\circ} \mathrm{C}$. Aliquots of supernatants and pellets were subjected to sodium dodecyl sulfate-polyacrylamide gel electrophoresis (SDS-PAGE) for detection of cytochrome $c$ release. Proteins were separated on a $15 \%$ gel and transferred onto nitrocellulose membranes. The immunoblots were treated with $15 \% \mathrm{H}_{2} \mathrm{O}_{2}$ for $15 \mathrm{~min}$ at room temperature and then sequentially incubated with $5 \%$ milk blocking solution, primary monoclonal antibody to cytochrome $c$ (PharMingen, San Diego, CA, USA) at a dilution of 1:5000 overnight at $4{ }^{\circ} \mathrm{C}$, and finally with secondary goat anti-mouse IgG antibody conjugated with horseradish peroxidase for $3 \mathrm{~h}$ at room temperature. Membranes were processed for cytochrome $c$ detection using the enhanced chemiluminescence light (ECL) reagent (Amersham Life Science, Inc., Arlington Heights, IL, USA).

\subsection{Densitometry and statistical analysis}

Densitometry was accomplished using a PC coupled to a PRIMAX ${ }^{\mathrm{TM}}$ 9600 Profi VM6575 scanner (Primax International B.V., Utrecht, The Netherlands). Quantitation of the autoradiograms used the ImageMaster 1D 
Elite densitometric analysis program (Amersham Pharmacia Biotech, Uppsala, Sweden). Fold and percent changes both in EPR parameters and protein levels were calculated based on corresponding controls. Differences between groups were compared using the unpaired two-tailed Student's $t$ test, performed on the basis of equal or unequal variance as appropriate, and $P$ values lower than 0.05 were considered statistically significant.

\section{Results}

3.1. Increased lipid polarity and fluidity sensed by the 7$D S A$ spin label reflect superficial interaction of $U C B$ with isolated mitochondrial membranes

To investigate the influence of UCB on mitochondrial membrane polarity at varying depths, we used 5-, 7-, and 16-DSA spin labels. Interaction of UCB with mitochondria resulted in decreased membrane polarity at a very superficial level, indicated by the decline in $a_{0}$ sensed by 5-DSA (Fig. 1A). In contrast, using the 7-DSA spin label, UCB caused a marked, dose-dependent increase in membrane polarity, which was more evident for lower UCB concentrations. Deeper in the membrane leaflet, $a_{0}$ was only slightly elevated as revealed by the 16-DSA probe. Thus, UCB decreases membrane polarity at the membrane-water interface, while promoting increased permeability in more hydrophobic regions. When $a_{0}$ was expressed as percentage of control, more pronounced alterations were detected at C-5 and C-7, whereas only a slight increase in membrane polarity was evident at C-16, greatly suggesting a superficial interaction (Fig. 1B). Finally, UCB also increased membrane order in the local microenvironment reported by 5-DSA. Using the 7DSA spin label, however, UCB markedly enhanced membrane fluidity. Expressing the motion parameters $\Delta l$ and $h_{+1} / h_{0}$ as percentage of control, a $5 \%$ increase in order was evident at C-5, while 9 and $2 \%$ decreases were detected at $\mathrm{C}-7$ and $\mathrm{C}-16$, respectively, corroborating the information given by the parameter $a_{0}$.

In contrast to the marked effects of UCB at C-7, ursodeoxycholate and cyclosporine $\mathrm{A}$ alone marginally altered the dynamic properties of mitochondrial membranes (Fig. 2A). However, both agents prevented UCB-induced alterations. In fact, pre-treatment with ursodeoxycholate inhibited the increase in mitochondrial membrane polarity caused by UCB by almost $75 \%(P<0.05)$, while cyclosporine A was equally protective.

\subsection{Disrupted protein order sensed by the 4-maleimido- TEMPO spin label reflects mitochondrial membrane instability induced by $U C B$}

Using the protein-oriented spin label 4-maleimidoTEMPO, the mobility parameter $h_{0} / h_{-1}$ is definitely decreased after exposure to $8.6 \mu \mathrm{M}$ UCB $(P<0.001)$, reflecting increased mobility of the probe and disrupted order structure of mitochondrial membrane proteins at the surface level (Fig. 2B). In contrast, ursodeoxycholate alone markedly increased protein order $(P<0.001)$. Pre-treat- ment with ursodeoxycholate completely abrogated UCBinduced effects, increasing protein order above controls $(P<0.001)$. These data suggests that stabilisation of the mitochondrial membrane by ursodeoxycholate may explain its ubiquitous protective role. Cyclosporine A alone did not alter protein mobility compared with controls but inhibited UCB-mediated perturbation $(P<0.05)$.

\subsection{Cytochrome c release induced by UCB reflects increased permeability in isolated mitochondrial membranes}

We then determined whether permeabilisation of the
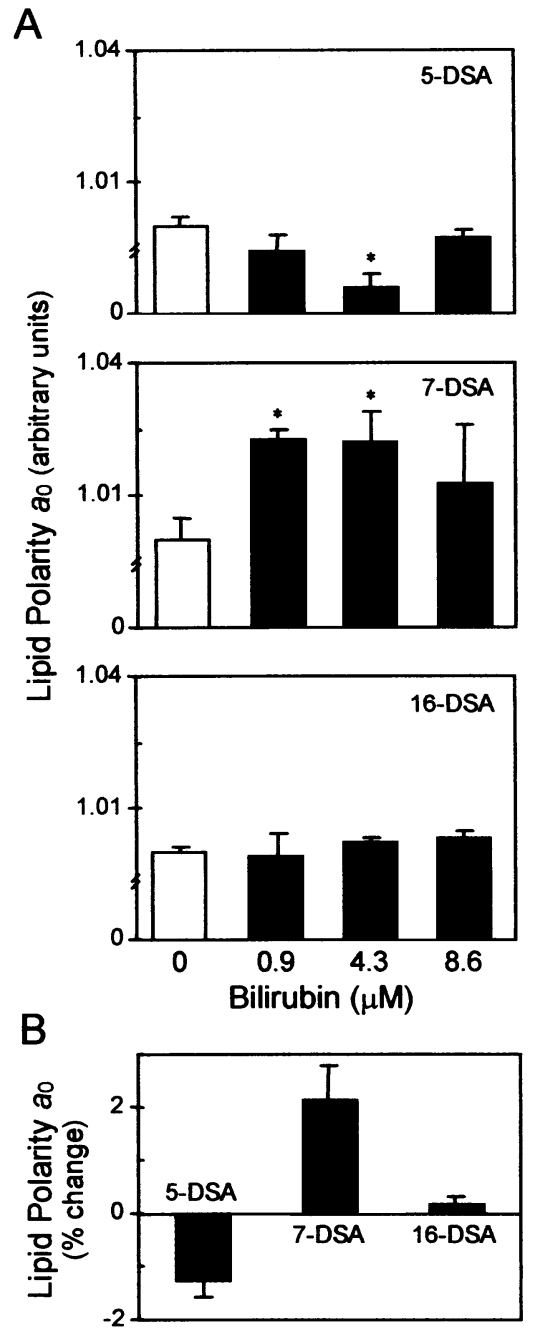

Fig. 1. UCB influences lipid polarity in rat mitochondrial membranes. Mitochondria were labelled with either 5-, 7-, or 16-DSA spin probes and then exposed to UCB in respiration buffer as described in Section 2. Mitochondrial pellets were examined for lipid polarity by EPR spectroscopy analysis. (A) Dose-response to UCB-induced alteration in mitochondrial membrane isotropic hyperfine splitting constant $\left(a_{0}\right)$. Labelled mitochondria were incubated with either UCB $(0.9,4.3$, and $8.6 \mu M)$ or no addition $(0 \mu \mathrm{M}$; control) for $5 \mathrm{~min}$. (B) Percent change in lipid polarity induced by $4.3 \mu \mathrm{M}$ UCB through the depth of the mitochondrial membrane leaflet. Values are mean \pm SEM relative to controls of at least three separate experiments. $* P<0.01$ from control. 
A

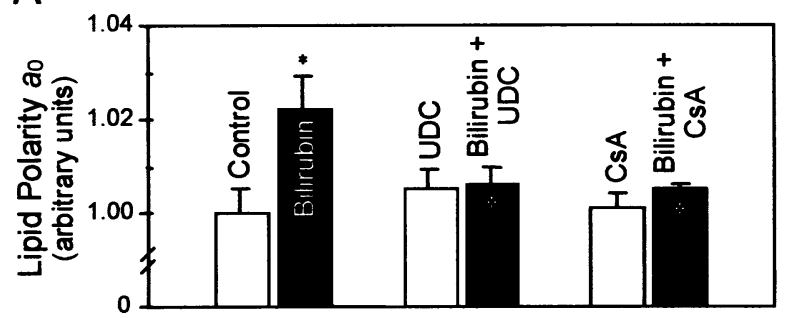

B

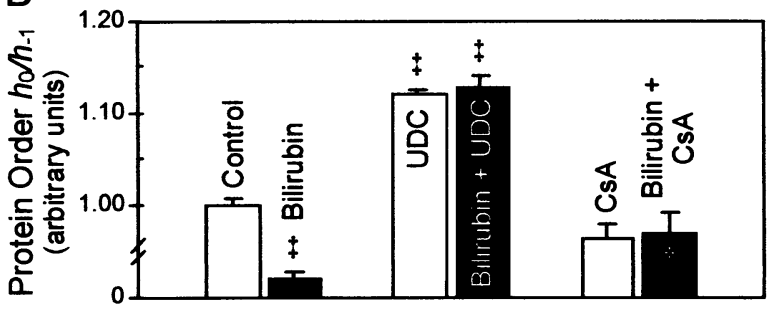

Fig. 2. Ursodeoxycholate and cyclosporine A abrogate disruption of lipid polarity and protein order induced by UCB in rat mitochondrial membranes. Labelled mitochondria were exposed to either $4.3 \mu \mathrm{M}$ $\mathrm{UCB}, 500 \mu \mathrm{M}$ ursodeoxycholate (UDC), $5 \mu \mathrm{M}$ cyclosporine A (CsA), UCB plus UDC, UCB plus CsA, or no addition ( $0 \mu \mathrm{M}$; control), for 5 min, in respiration buffer as described in Section 2. In co-incubation experiments, mitochondria were pre-treated with either UDC or CsA for $5 \mathrm{~min}$. Mitochondrial pellets were examined for lipid polarity and protein order by EPR spectroscopy analysis. (A) Alteration in mitochondrial membrane isotropic hyperfine splitting constant $\left(a_{0}\right)$ sensed by the 7-DSA spin label. (B) Alteration in the ratio of the height of the midline to the height of the high-field line $\left(h_{0} / h_{-1}\right)$ sensed by the 4maleimido-TEMPO spin label. Values are mean \pm SEM relative to controls of at least three separate experiments. $\dagger P<0.05$ from UCB alone; $* P<0.01$ from control; $\ddagger P<0.001$ from control and UCB alone.

mitochondrial membrane by direct interaction with UCB was accompanied by the release of intermembrane proteins, such as cytochrome $c$. Levels of cytochrome $c$ in supernatants of controls were undetectable and, likewise, addition of either ursodeoxycholate or cyclosporine A did not significantly influence cytochrome $c$ release. In contrast, mitochondria treated with UCB showed marked, dosedependent release of cytochrome $c$, while supernatant levels were proportionally increased (Fig. 3, top). Pre-incubation with either ursodeoxycholate or cyclosporine A prevented efflux of cytochrome $c$ by 30-60\% $(P<0.05)$ (Fig. 3, bottom). Thus, interaction of UCB with mitochondrial membranes results in cytochrome $c$ redistribution, which appears to be mediated, at least in part, via the mitochondrial permeability transition pore opening.

\subsection{Loss of spin-label intensity induced by UCB reflects oxidative injury in isolated mitochondrial membranes}

To determine if exposure to UCB modifies levels of mitochondrial membrane oxidation, isolated mitochondria were probed with 5- and 12-DSA spin labels and then exposed to UCB. UCB caused a rapid, dose-dependent loss of spinprobe intensity, manifested by a decrease in peak amplitude
(Fig. 4A). Treatment with $0.9 \mu \mathrm{M}$ UCB for $5 \mathrm{~min}$ did not significantly alter 5-DSA signal amplitude, while $4.3 \mu \mathrm{M}$ concentrations caused a $14 \%$ decrease in spin-probe intensity $(P<0.01)$. Decreases in signal amplitude were slightly more marked at $15 \mathrm{~min}$ of incubation, but remained unchanged for a subsequent 60-min incubation period (data not shown). The magnitude of the 12-DSA signal incorporated into mitochondria membranes was hardly decreased during exposure to $\mathrm{UCB}$, indicative of less damage in deeper regions of the bilayer. Using a different methodological approach to measure signal intensity, based on the double integration of spectra, we confirmed the results described above. In fact, $4.3 \mu \mathrm{M}$ UCB led to an almost $20 \%$ decrease of the 5-DSA signal amplitude as compared to controls, while the 12-DSA signal was only marginally affected.

Spin-label signal amplitude in isolated mitochondria was also assessed in the presence of ursodeoxycholate (Fig. 4B). Treatment of 5-DSA-labelled mitochondria with the bile acid alone did not significantly decrease signal amplitude, while incubation with ursodeoxycholate for $5 \mathrm{~min}$ prior to exposure to $4.3 \mu \mathrm{M}$ UCB markedly inhibited oxidative injury. This protection resulted in signal amplitudes that were no different from controls, indicating that ursodeoxycholate either confers direct protection against lipid peroxidation in isolated mitochondrial membranes or prevents UCB insertion into the membrane indirectly inhibiting reactive oxygen species production.

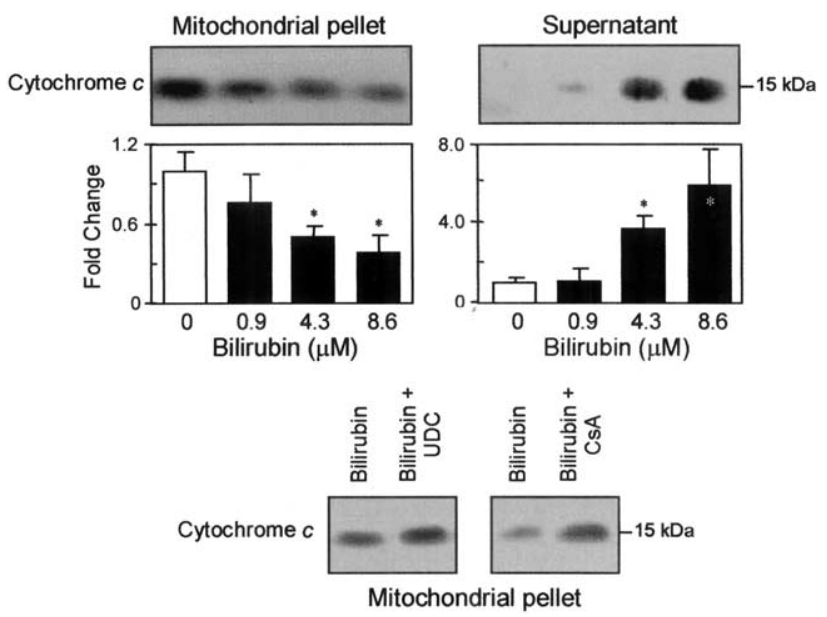

Fig. 3. Dose-response of isolated mitochondria to UCB-induced cytochrome $c$ release and protection by ursodeoxycholate and cyclosporine A. Mitochondria were incubated with either UCB (0.9, 4.3, and 8.6 $\mu \mathrm{M}$ ), 4.3 $\mu \mathrm{M}$ UCB plus $500 \mu \mathrm{M}$ ursodeoxycholate (UDC), $4.3 \mu \mathrm{M}$ UCB plus $5 \mu$ M cyclosporine A (CsA), or no addition ( $0 \mu M$; control), for $5 \mathrm{~min}$, in respiration buffer as described in Section 2. Mitochondrial pellets and supernatants were examined for cytochrome $c$ levels by Western blot analysis. Following SDS-PAGE and transfer, the nitrocellulose membranes were incubated with the monoclonal antibody to cytochrome $c$ and the 15-kDa protein was detected using ECL chemiluminescence. Blots are representative of at least three separate experiments and the accompanying histograms are the densitometric means \pm SEM relative to controls. $* P<0.01$ from control. 

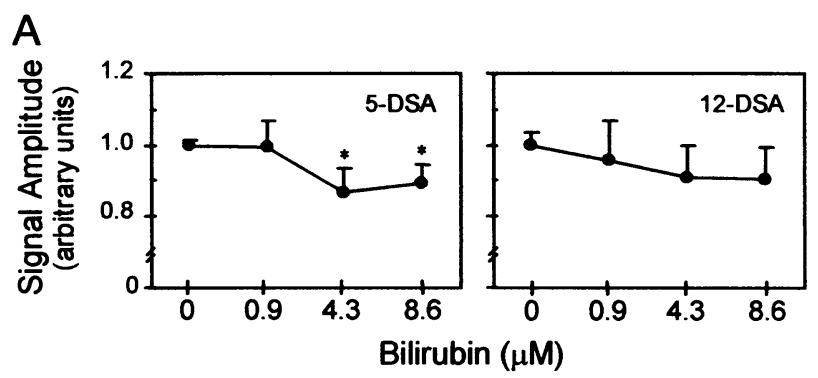

B

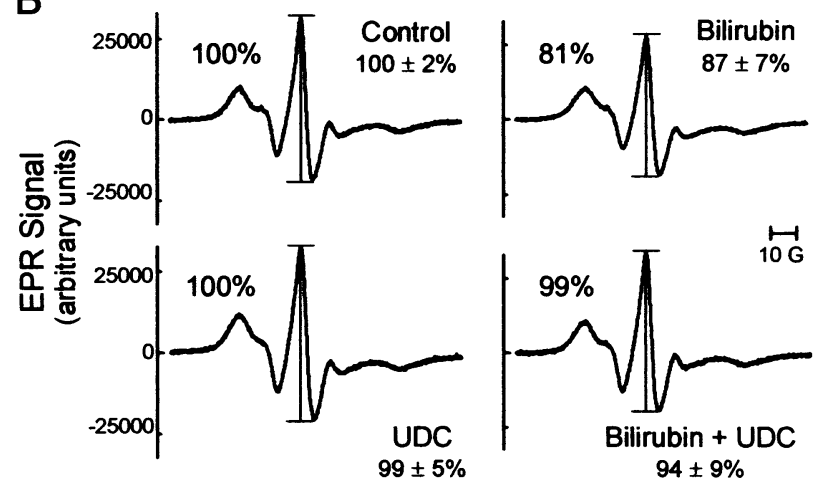

Fig. 4. Ursodeoxycholate prevents oxidative injury induced by UCB in mitochondrial membranes. Mitochondria were labelled with either 5or 12-DSA spin probes and then exposed to UCB in respiration buffer as described in Section 2. Mitochondrial pellets were examined for reactive oxygen species production by EPR spectroscopy analysis. (A) Dose-response to UCB-induced decrease in signal amplitude sensed by 5- and 12-DSA spin labels. Labelled mitochondria were incubated with either UCB $(0.9,4.3$, and $8.6 \mu M)$ or no addition $(0 \mu \mathrm{M}$; control) for 5 min. (B) Alteration in EPR spectra sensed by the 5-DSA spin label. Labelled mitochondria were exposed to either $4.3 \mu \mathrm{M}$ UCB, $500 \mu \mathrm{M}$ ursodeoxycholate (UDC), UCB plus UDC, or no addition (control) for 5 min. In co-incubation experiments, mitochondria were pre-treated with UDC for $5 \mathrm{~min}$. Spectra are representative of at least three separate experiments and values are mean \pm SEM relative to controls. $* \boldsymbol{P}<0.01$ from control.

\section{Discussion}

The present study demonstrates direct physical interaction of UCB with isolated rat mitochondrial membranes, wherein it acts to disrupt lipid polarity and fluidity, protein order, and redox status. These novel findings, when taken together with our prior studies showing morphologic characteristics of apoptosis in cultured nerve cells exposed to UCB $[12,13]$, suggest that UCB-induced cytotoxicity may be mediated, in part, by its effect of physically perturbing the mitochondrial membrane structure.

The 'physiologic jaundice' of neonates is often an innocuous condition, which conceivably represents a transitional antioxidative mechanism in the neonatal circulation $[39,40]$. However, high concentrations of UCB can be neurotoxic and, therefore, neonatal hyperbilirubinemia may exceptionally result in death, or survival with severe neurological sequelae [41]. Evidence indicates that UCB toxicity may be a consequence of disrupted mitochondrial function $[12,14-18]$.
The present study suggests that UCB intercalates at C-5, increasing phospholipid packing and thus reducing the already low mobility of the phospholipid-acyl chain at this region of the leaflet. Such a superficial effect renders inner regions more permeable, as confirmed by the increased polarity and fluidity at $\mathrm{C}-7$ and $\mathrm{C}-16$. Moreover, since perturbation of the mitochondrial membrane induced by UCB decreases from C-7 to C-16, it is conceivable that this gradient results from a secondary effect to the superficial accommodation of UCB. This scenario, although in agreement with results obtained using an erythrocyte model [42], does not exclude a possible interaction of UCB with even more external regions of the leaflet. To this end, kinetic and thermodynamic studies on the movement of UCB between intracellular membranes have suggested that UCB is associated with phospholipid bilayers at the membrane-water interface [43]. Moreover, the solubility characteristics of the molecule would not favour its intercalation between the phospholipid acyl chain [44]. The increased permeability of the mitochondrial membrane induced by UCB as described above is also supported by the observation that UCB augments the mobility of the 4maleimido-TEMPO spin label, indicating disruption of the protein order structure and increased membrane fluidity much above control values. As a final note, UCB appears to interact with mitochondrial membrane lipids in a fashion similar to that described in a recent study using erythrocyte membranes but, nevertheless, higher UCB concentrations [42], thus suggesting a more generic perturbation.

In contrast to the effects of UCB, ursodeoxycholate appears not to damage mitochondrial membranes but when combined with the pigment almost completely inhibits mitochondrial membrane instability. Further, using the taurine conjugated derivative of ursodeoxycholate, a related hydrophilic bile salt, we also detected significant protection against UCB-induced perturbation (data not shown). The stabilisation of the mitochondrial membrane by ursodeoxycholate reported in this study, and likewise suggested by previously published data [19-21], provides an additional explanation for its protective role at preventing apoptosis in several model systems. Finally, we did not detect any major disruption of lipid or protein surface structure induced by cyclosporine A alone. This is in agreement with the matrix location of cyclophilin $\mathrm{D}$, the mitochondrial target of cyclosporine A. However, cyclosporine A, abrogated the effects of UCB on membrane structure. Thus, these results although not excluding a direct physicochemical interaction between UCB and cyclsoporine A, are consistent with a protection that occurs via the inhibition of the permeability transition pore.

The present study also demonstrates that cytochrome $c$ is released from isolated rat mitochondria following superficial insertion of UCB, and subsequent disruption of membrane lipid polarity and protein order. Cytochrome $c$ redistribution is a common event in the cell death effector pathway initiated by diverse apoptotic stimuli [45-47], even 
preceding exposure of phosphatidylserine and loss of plasma-membrane integrity in apoptotic cells [48]. Nevertheless, the precise mechanism of cytochrome $c$ efflux and its regulation remain a matter of debate. Several reports have provided evidence that redistribution occurs independently of any detectable mitochondrial depolarization, implying that opening of the megapore is a downstream event to caspase activation [49]. However, data presented here suggests that interaction of UCB with the mitochondrial membrane increases permeability, which in turn is required for cytochrome $c$ release. This is in agreement with previous work, where mitochondrial permeabilisation was shown to coincide with cytochrome $c$ relocation, and precede caspase activation [20,50,51]. Moreover, co-incubation of mitochondria with ursodeoxycholate or cyclosporine A resulted in significant inhibition of mitochondrial permeability transition induced by UCB and, therefore, prevented cytochrome $c$ release.

Probably as a consequence of cytochrome $c$ release, the paramagnetism of both 5- and 12-DSA was decreased in mitochondria incubated with UCB. Concentrations of $\mathrm{UCB}$ in the low $\mu \mathrm{M}$ range induced membrane lipid peroxidation by a mechanism that appears to involve direct interaction of UCB with the mitochondrial membrane with subsequent release of cytochrome $c$, and then disruption of the respiratory chain. Alternatively, reactive oxygen species production may cause dissulfide formation in the pore structure and open channels to release intermembrane proteins. The release of cytochrome $c$ may further inhibit the transfer of electrons in the respiratory chain, again increasing oxidative stress, in a vicious cycle.

In conclusion, our data indicate that the superficial interaction of UCB with the mitochondrial membrane likely accounts for the disruption of lipid membrane polarity and fluidity, and altered protein order induced by UCB. The increased membrane permeability sensed by spin-label EPR analysis is consistent with the reduced levels of cytochrome $c$ found in mitochondria, and can then lead to subsequent membrane lipid peroxidation. Thus, the results suggest that UCB-induced cytotoxicity is mediated, at least in part, by its effect of physically perturbing the mitochondrial membrane. These novel findings may ultimately prove useful to our evolving understanding of UCB cytotoxicity.

\section{Acknowledgements}

The authors thank members of the laboratory for their comments during the course of this work; and Carlos Brondino from the Departamento de Química, FCT, Universidade Nova de Lisboa, Monte de Caparica, Portugal for his expertise in the use of the electron paramagnetic resonance spectrometer. This work was supported in part by grant PRAXIS/C/SAU/14311/1998 from Fundação para a Ciência e a Tecnologia, Lisbon, Portugal and by a Research Fellow- ship from the European Association for the Study of the Liver (to C.M.P.R.).

\section{References}

[1] Brodersen R, Stern L. Deposition of bilirubin acid in the central nervous system - a hypothesis for the development of kernicterus. Acta Paediatr Scand 1990;79:12-19.

[2] Rubboli G, Ronchi F, Cecchi P, Rizzi R, Gardella E, Meletti S, et al. A neurophysiological study in children and adolescents with CriglerNajjar syndrome type I. Neuropediatrics 1997;28:281-286.

[3] Majumdar APN. Bilirubin encephalopathy: effect on RNA polymerase activity and chromatin template activity in the brain of Gunn rat. Neurobiology 1974;4:425-431.

[4] Yamada N, Sawasaki Y, Nakajima H. Impairment of DNA synthesis in Gunn rat cerebellum. Brain Res 1977;126:295-307.

[5] Ohno T. Kernicterus: effect on choline acetyltransferase, glutamic acid decarboxylase and tyrosine hydroxylase activities in the brain of Gunn rat. Brain Res 1980;196:282-285.

[6] Morphis L, Constantopoulos A, Matsaniotis N, Papaphilis A. Bilirubin-induced modulation of cerebral protein phosphorylation in neonate rabbits in vivo. Science 1982;218:156-158.

[7] Sano K, Nakamura H, Matsuo T. Mode of inhibitory action of bilirubin on protein kinase C. Pediatr Res 1985;19:587-590.

[8] Schiff D, Chan G, Poznansky MJ. Bilirubin toxicity in neuronal cell lines N115 and NBR10A. Pediatr Res 1985;19:908-911.

[9] Hansen TWR, Bratlid D, Walaas SI. Bilirubin decreases phosphorylation of synapsin I, a synaptic vesicle-associated neuronal phosphoprotein, in intact synaptosomes from rat cerebral cortex. Pediatr Res 1988;23:219-223.

[10] Silva R, Mata LR, Gulbenkian S, Brito MA, Tiribelli C, Brites D. Inhibition of glutamate uptake by unconjugated bilirubin in cultured cortical rat astrocytes: role of concentration and $\mathrm{pH}$. Biochem Biophys Res Commun 1999;265:67-72.

[11] Grojean S, Koziel V, Vert P, Daval JL. Bilirubin induces apoptosis via activation of NMDA receptors in developing rat brain neurons. Exp Neurol 2000;166:334-341.

[12] Rodrigues CMP, Solá S, Silva R, Brites D. Bilirubin and amlyloid- $\beta$ peptide induce cytochrome $c$ release through mitochondrial membrane permeabilization. Mol Med 2000;6:936-946.

[13] Silva R, Rodrigues CMP, Brites D. Bilirubin-induced apoptosis in glial and nerve cells is aggravated by chenodeoxycholic acid but prevented by ursodeoxycholic acid. J Hepatol 2001;34:402-408.

[14] Cowger ML, Igo RP, Labbe RF. The mechanism of bilirubin toxicity studied with purified respiratory enzyme and tissue culture systems. Biochemistry 1965;4:2763-2770.

[15] Mustafa MG, Cowger ML, King TE. Effects of bilirubin on mitochondrial reactions. J Biol Chem 1969;244:6403-6414.

[16] Noir BA, Boveris A, Garaza Pereira AM, Stoppani AO. Bilirubin: a multi-site inhibitor of mitochondrial respiration. FEBS Lett 1972;27:270-274.

[17] Batty HK, Millhouse OE. Ultrastructure of the Gunn rat substantia nigra. II. Mitochondrial changes. Acta Neuropathol 1976;34:7-19.

[18] Mayor Jr F, Diez-Guerra J, Valdivieso F, Mayor F. Effect of bilirubin on the membrane potential of rat brain synaptosomes. J Neurochem 1986;47:363-369.

[19] Rodrigues CMP, Fan G, Ma X, Kren BT, Steer CJ. A novel role of ursodeoxycholic acid in inhibiting apoptosis by modulating mitochondrial membrane perturbation. J Clin Invest 1998;101:2790-2799.

[20] Rodrigues CMP, Ma X, Linehan-Stieers C, Fan G, Kren BT, Steer CJ. Ursodeoxycholic acid prevents cytochrome $c$ release in apoptosis by inhibiting mitochondrial membrane depolarization and channel formation. Cell Death Differ 1999;6:842-854.

[21] Rodrigues CMP, Linehan-Stieers C, Keene CD, Ma X, Low W, Kren BT, et al. Tauroursodeoxycholic acid partially prevents apoptosis 
induced by 3-nitropropionic acid: evidence for a mitochondrial pathway independent of the permeability transition. J Neurochem 2000;75:2368-2379.

[22] Thornberry NA, Lazebnik Y. Caspases: enemies within. Science 1998;281:1312-1316.

[23] Green DR, Reed JC. Mitochondria and apoptosis. Science 1998;281:1309-1312.

[24] Walajtys-Rhode E, Zapatero J, Moehren G, Hoek JB. The role of the matrix calcium level in the enhancement of mitochondrial pyruvate carboxylation by glucagon pretreatment. J Biol Chem 1992;267:370379.

[25] Botla R, Spivey JR, Aguilar H, Bronk SF, Gores GJ. Ursodeoxycholate (UDCA) inhibits the mitochondrial membrane permeability transition induced by glycochenodeoxycholate: a mechanism of UDCA cytoprotection. J Pharmacol Exp Ther 1995;272:930-938.

[26] Rodrigues CMP, Fan G, Wong PY, Kren BT, Steer CJ. Ursodeoxycholic acid may inhibit deoxycholic acid-induced apoptosis by modulating mitochondrial transmembrane potential and reactive oxygen species production. Mol Med 1998;4:165-178.

[27] Jost P, Libertini LJ, Hebert VC, Griffith OH. Lipid spin labels in lecithin multilayers. A study of motion along fatty acid chains. J Mol Biol 1971;59:77-98.

[28] Harris J, Power TJ, Bieber AL, Watts A. An electron-spin-resonance spin-label study of the interaction of purified Mojave toxin with synaptosomal membranes from rat brain. Eur $\mathrm{J}$ Biochem 1983;131:559-565.

[29] Güldütuna S, Zimmer G, Leuschner M, Bhatti S, Elze A, Deisinger B, et al. The effect of bile salts and calcium on isolated rat liver mitochondria. Biochim Biophys Acta 1999;1453:396-406.

[30] Schreier S, Frezzatti WA, Araujo PS, Chaimovich H, Cuccovia IM. Effect of lipid membranes on the apparent pK of the local anesthetic tetracaine. Spin label and titration studies. Biochim Biophys Acta 1984;769:231-237.

[31] Bianconi ML, do Amaral AT, Schreier S. Use of membrane spin label spectra to monitor rates of reaction of partitioning compounds: hydrolysis of a local anesthetic analog. Biochem Biophys Res Commun 1988;152:344-350.

[32] Lai CS, Tooney NM, Ankel EG. Structure and flexibility of plasma fibronectin in solution: electron spin resonance spin-label, circular dichroism, and sedimentation studies. Biochemistry 1984;23:63936397.

[33] Kunicki TJ, Nugent DJ, Piotrowicz RS, Lai CS. Covalent attachment of sulfhydryl-specific, electron spin resonance spin-labels to Fab' fragments of murine monoclonal antibodies that recognize human platelet membrane glycoproteins. Development of membrane protein specific spin probes. Biochemistry 1986;25:4979-4983.

[34] Butterfield DA, Hensley K, Harris M, Mattson M, Carney J. $\beta$ Amyloid peptide free radical fragments initiate synaptosomal lipoperoxidation in a sequence specific fashion: implications to Alzheimer's disease. Biochem Biophys Res Commun 1994;200:710-715.

[35] Hensley K, Carney J, Hall N, Shaw W, Butterfield DA. Electron paramagnetic resonance investigations of free radical-induced altera- tions in neocortical synaptosomal membrane protein infrastructure. Free Radic Biol Med 1994;17:321-331.

[36] Bruce-Keller AJ, Begley JG, Fu W, Butterfield DA, Bredesen DE, Hutchins JB, et al. Bcl-2 protects isolated plasma and mitochondrial membranes against lipid peroxidation induced by hydrogen peroxide and amyloid $\beta$-peptide. J Neurochem 1998;70:31-39.

[37] Blanckaert N, Heirwegh KPM. Analysis and preparation of bilirubins and biliverdins. In: Ostrow JD, editor. Bile Pigments and Jaundice, New York: Marcel Dekker, 1986. pp. 31-79.

[38] McDonagh AF, Assisi F. The ready isomerization of bilirubin IX- in aqueous solution. Biochem J 1972;129:797-800.

[39] Dennery PA, Rodgers PA. Ontogeny and developmental regulation of heme oxygenase. J Perinatol 1996;16:S79-S83.

[40] Bélanger S, Lavoie JC, Chessex P. Influence of bilirubin on the antioxidant capacity of plasma in newborn infants. Biol Neonate 1997;71:233-238.

[41] Gourley GR. Bilirubin metabolism and kernicterus. Adv Pediatr 1997;44:173-229.

[42] Brito MA, Brondino CD, Moura JJG, Brites D. Effects of bilirubin molecular species on membrane dynamic properties of human erythrocyte membranes. A spin-label electron paramagnetic resonance spectroscopy study. Arch Biochem Biophys 2001;387:57-65.

[43] Zucker SD, Storch J, Zeidel ML, Gollan JL. Mechanism of the spontaneous transfer of unconjugated bilirubin between small unilamellar phosphatidylcholine vesicles. Biochemistry 1992;31:3184-3192.

[44] Brodersen R. Bilirubin. Solubility and interaction with albumin and phospholipid. J Biol Chem 1979;254:2364-2369.

[45] Kim CN, Wang X, Huang Y, Ibrado AM, Liu L, Fang G, et al. Overexpression of $\mathrm{Bcl}-\mathrm{X}_{\mathrm{L}}$ inhibits Ara-C-induced mitochondrial loss of cytochrome $c$ and other perturbations that activate the molecular cascade of apoptosis. Cancer Res 1997;57:3115-3120.

[46] Kluck RM, Bossy-Wetzel E, Green DR, Newmeyer DD. The release of cytochrome $c$ from mitochondria: a primary site for Bcl-2 regulation of apoptosis. Science 1997;275:1132-1136.

[47] Yang J, Liu X, Bhalla K, Kim CN, Ibrado AM, Cai J, et al. Prevention of apoptosis by Bcl-2: release of cytochrome $c$ from mitochondria blocked. Science 1997;275:1129-1132.

[48] Goldstein JC, Waterhouse NJ, Juin P, Evan GI, Green DR. The coordinate release of cytochrome $c$ during apoptosis is rapid, complete and kinetically invariant. Nat Cell Biol 2000;2:156-162.

[49] Bossy-Wetzel E, Newmeyer DD, Green DR. Mitochondrial cytochrome $c$ release in apoptosis occurs upstream of DEVD-specific caspase activation and independently of mitochondrial transmembrane depolarization. EMBO J 1998;17:37-49.

[50] Liu X, Kim CN, Yang J, Jemmerson R, Wang X. Induction of apoptotic program in cell-free extracts: requirement for dATP and cytochrome $c$. Cell 1996;86:147-157.

[51] Li P, Nijhawan D, Budihardjo I, Srinivasula SM, Ahmad M, Alnemri ES, et al. Cytochrome $c$ and dATP-dependent formation of Apaf-1/ caspase- 9 complex initiates an apoptotic protease cascade. Cell 1997;91:479-489. 